la revue La revue pour l'histoire du CNRS

POUR L'HISTOIRE DU CNRS $\quad 8$ | 2003

Aux origines de l'Homme

Histoire secrète de la bombe atomique française

André Bendjebbar. Le Cherche Midi Éditeur, Paris, 2000

Jean-Claude Allain

(2) OpenEdition

Journals

Édition électronique

URL : https://journals.openedition.org/histoire-cnrs/408

DOI : $10.4000 /$ histoire-cnrs.408

ISSN : 1955-2408

Éditeur

CNRS Éditions

Édition imprimée

Date de publication : 5 mai 2003

ISBN : 978-2-271-06068-6

ISSN : $1298-9800$

Référence électronique

Jean-Claude Allain, « Histoire secrète de la bombe atomique française », La revue pour l'histoire du CNRS [En ligne], 8| 2003, mis en ligne le 07 mars 2006, consulté le 20 mai 2021. URL : http://

journals.openedition.org/histoire-cnrs/408; DOI : https://doi.org/10.4000/histoire-cnrs.408

Ce document a été généré automatiquement le 20 mai 2021

Comité pour l'histoire du CNRS 


\section{Histoire secrète de la bombe atomique française}

André Bendjebbar. Le Cherche Midi Éditeur, Paris, 2000

Jean-Claude Allain

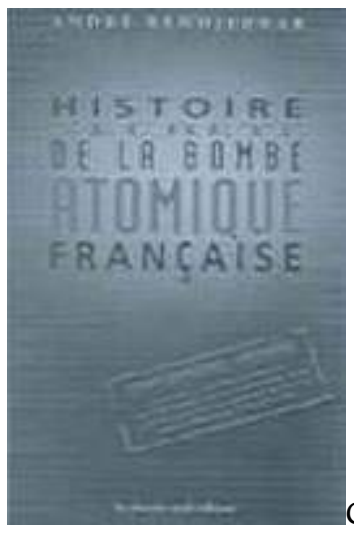

Cet ouvrage est la version éditée d'une thèse soutenue à l'Institut d'études politiques de Paris en 2000 sous un titre moins médiatique ( La bombe atomique et deux Républiques-1939-1969») et présentant l'histoire de l'accession de la France à l'arme nucléaire. On en connaissait de multiples aspects par les récits en forme de témoignages de certains de ses acteurs (comme le chimiste Bertrand Goldschmidt, le général Albert Buchalet, le professeur Yves Rocard, le ministre Pierre Messmer) et par des études historiques de la dernière décennie (notamment par Dominique Mongin ou sous la direction de Maurice Vaïsse). On percevait le profil d'ensemble: la contribution des scientifiques à la fin des années 1930 , le cheminement camouflé et contesté de "l'aventure nucléaire » sous la IV République, sa promotion "ostentatoire " (A. Bendjebbar) au début de la République gaullienne. À cette vision floue et en mosaïque, André Bendjebbar substitue une analyse suivie et coordonnée, reprenant et confrontant les divers récits connus, exploitant les archives ouvertes des National Archives et du Quai d'Orsay, celles des fonds Frédéric Joliot, Pierre Mendès-France, etc., et des interviews de scientifiques, de politiques, de militaires. Le traitement de cette immense collecte a donné cette remarquable étude sur la bombe A française jusqu'aux années 1960-1962, à laquelle 22 chapitres sur 25 sont 
consacrés, la partie concernant la bombe $\mathrm{H}$, n'étant, à cette aune, qu'esquissée, non sans révélations $\mathrm{du}$ reste (une proposition britannique d'échanger une aide technologique contre l'entrée dans la Communauté économique européenne).

La problématique de l'ouvrage s'articule autour de la relation, dans le domaine sensible de la défense, entre le milieu scientifique et l'autorité politique qui n'ont pas nécessairement la même approche, ni des vues convergentes, surtout quand le pouvoir politique traverse une phase critique (1940-1944) ou s'exerce difficilement (1947-1958). L'objectif scientifique des savants est la découverte, mais aussi sa valorisation dans leur milieu national et international, d'où le dépôt de brevets, comme le dit F. Joliot en 1939 (et dont la rocambolesque histoire nous est ici contée), une publicité qui s'accorde mal avec le «secret défense ». La personnalité de F. Joliot est au cœur du premier tiers de l'ouvrage, jusqu'à sa révocation en 1950.

2 André Bedjebbar s'emploie à en éclairer et expliquer les manifestations déconcertantes, en 1940 par exemple, quand il choisit de rester en France après avoir envoyé ses collaborateurs (avec ses brevets) en Angleterre et mis à l'abri les stocks d'eau lourde et de sels d'uranium, ceux-ci au Maroc (d'où on ne les voit pas revenir de Taroudant après la guerre). Le savant, qui a son réseau international de collègues et d'élèves, y compris allemands (l'amitié de Wolfgang Gentner sera bien utile pendant l'Occupation), se révèle aussi un véritable manageur, car il ne dissocie pas la recherche fondamentale de ses applications, en relation d'affaires avec tout un réseau d'entreprises industrielles. Après avoir promis au général de Gaulle de lui «faire [sa] bombe », F. Joliot vire au pacifisme, à la grande satisfaction des Soviétiques qui ne se privent pas de l'exploiter mais cette conversion s'inscrit dans un vaste courant de contestation de l'arme atomique qui touche aussi bien les scientifiques américains qu'une large fraction de l'opinion publique française. En 1946, Alexandre Parodi présente à l'Organisation des nations unies la position officielle française qui prévaudra pendant quelques années : le choix du nucléaire civil et l'abandon de la filière militaire.

3 Néanmoins, pendant toute la IV République, la recherche sur la bombe se poursuit dans un clair-obscur nécessaire au secret défense et entretenu, délibérément ou tacitement, par opportunisme politique, par les chefs de gouvernement et les ministres compétents. Félix Gaillard est l'exception connue, depuis le plan de 1952 qui comporte des crédits pour la production de plutonium jusqu'à sa décision du 17 avril 1958 de faire procéder aux premiers essais en 1960, cette dernière est prise toutefois en catimini (et même en toute irrégularité). Les cas de P. Mendès France et de G. Mollet sont réexaminés pour montrer leur adhésion à cette acquisition de puissance nationale que sera la bombe, par revirement chez G. Mollet, avant même, semble-t-il, l'épisode de la sommation soviétique pendant la crise de Suez, par conviction plus profonde et soutenue chez P. Mendès France, qui agit pour sauvegarder la souveraineté atomique de la France, en laissant rejeter la Communauté européenne de défense, CDE, (on pourrait rappeler qu'il négocie sa reconversion en Union de l'Europe occidentale avec les Britanniques), puis en surveillant la négociation du traité sur l'Euratom. L'arrivée de De Gaulle change le mode de gestion du dossier nucléaire, en l'assumant publiquement urbi et orbi jusqu'au premier essai de Gerboise bleue le 13 février 1960 : un pari politique et diplomatique gagné mais qui fut bien risqué, quand on sait, de juillet 1958 jusqu'à la veille de l'essai, les incertitudes techniques qui restaient à résoudre et les interrogations météorologiques sur les courants aériens de l'atmosphère saharienne. 
$4 \mathrm{Au}$ fil de tous ces épisodes, A. Bendjebbar met en relief deux aspects essentiels de la question : le rôle du « groupe » pro-nucléaire et la dimension internationale. La bombe est l'œuvre d'un de ces "petits groupes de dirigeants» de la catégorie « directionnelle » (cf. Relations internationales, $n^{\circ} 41,1985$ ), unissant pour la préparation semi-clandestine de la fabrication de la bombe des personnalités d'horizons divers mais partageant le même objectif: scientifiques civils et militaires, hauts fonctionnaires, responsables politiques, mais tous toujours en deçà du seuil décisionnel (exemple : Y. Rocard, Francis Perrin, Charles Ailleret, A. Buchalet, Pierre Guillaumat, M.-P. Koenig, F. Gaillard avant 1958). Ils constituent un réseau relationnel, informel mais bien réel, et non exempt de rivalités internes : entre civils du Commissariat à l'énergie atomique, CEA, et militaires, qui auraient, comme C. Ailleret, volontiers assumé la responsabilité de toute la chaine de production, entre corps et services différents, dont celui des Poudres, utilement récupéré ou rallié in extremis.

5 La dimension internationale est essentiellement franco-américaine, secondairement franco-britannique ou allemande ; son analyse doit beaucoup aux archives américaines. Les États-Unis sont hostiles à l'accession de la France à un armement nucléaire qu'elle ne leur devrait pas, et, avant la lettre, à une prolifération, contraire à l'accord anglosaxon de 1943 et déjà entamée avec les Russes. Scientifiquement et politiquement, comment la dissuader de s'engager dans cette voie? Elle a déjà un potentiel de savoir sur l'atome (même si on affecte d'ignorer souverainement la contribution initiale de ses savants) et la coopération des scientifiques civils comme les missions des " atomistes » militaires lui permettent de collecter des renseignements techniques utiles à la recherche, puis à la fabrication de la bombe, dont on pourra assurer qu'elle est purement de conception française. Sa réalisation devient inévitable, après l'échec du contre-feu de la CED, puis de l'Euratom, et surtout quand de Gaulle en fait un enjeu national. Il est alors trop tard pour offrir de la lui «donner» (D. D. Eisenhower, décembre 1959) et, ensuite, pour maîtriser l'incidence stratégique de ce troisième partenaire nucléaire, occidental mais se voulant autonome, pour lui proposer un partage de la décision nucléaire, dans le cadre de la Multilateral Force (J. F. Kennedy, 1963), partage décisionnel qu'on avait refusé en 1958. Entretemps, l'essai a eu lieu à Reggane -décrit dans le remarquable chapitre XVII-, et d'autres ont suivi au cours de l'année suivante. Naturellement, la planète entière a condamné ces essais et on en connaîtra une nouvelle édition en 1995 !

6 Fût-ce par l'appartenance de F. Joliot au premier CNRS, l'ouvrage d'A. Bendjebbar méritait une recension dans La Revue, mais c'est l'ensemble du récit, d'une lecture aisée, même dans ses passages techniques, pédagogiquement exposés, qui appelle de réels compliments à son auteur.

\section{INDEX}

Mots-clés : bombe, atomique, arme, nucléaire, militaire, archive, politique, pouvoir 
AUTEUR

JEAN-CLAUDE ALLAIN

Professeur émérite de l'université Paris III, Sorbonne nouvelle 